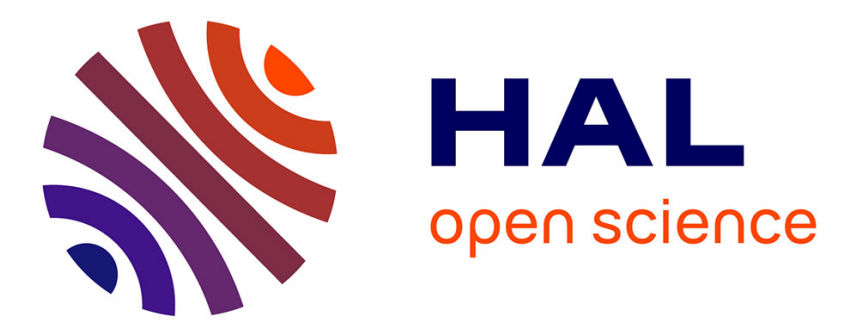

\title{
Road network emergency accessibility planning after a major earthquake
}

\author{
Celso Satoshi Sakuraba, Andréa Cynthia Santos, Christian Prins, Lucie \\ Bouillot, Arnaud Durand, Bernard Allenbach
}

\section{- To cite this version:}

Celso Satoshi Sakuraba, Andréa Cynthia Santos, Christian Prins, Lucie Bouillot, Arnaud Durand, et al.. Road network emergency accessibility planning after a major earthquake. EURO Journal on Computational Optimization, 2016, 4 (3-4), pp.381-402. 10.1007/s13675-016-0070-2 . hal-02291629

\section{HAL Id: hal-02291629}

\section{https://hal-utt.archives-ouvertes.fr/hal-02291629}

Submitted on 26 Oct 2020

HAL is a multi-disciplinary open access archive for the deposit and dissemination of scientific research documents, whether they are published or not. The documents may come from teaching and research institutions in France or abroad, or from public or private research centers.
L'archive ouverte pluridisciplinaire HAL, est destinée au dépôt et à la diffusion de documents scientifiques de niveau recherche, publiés ou non, émanant des établissements d'enseignement et de recherche français ou étrangers, des laboratoires publics ou privés. 


\title{
Road network emergency accessibility planning after a major earthquake
}

\author{
Celso Satoshi Sakuraba ${ }^{1,2}$ - Andréa Cynthia Santos ${ }^{2}$. \\ Christian Prins $^{2}$ - Lucie Bouillot ${ }^{3}$. Arnaud Durand ${ }^{3}$. \\ Bernard Allenbach ${ }^{3}$
}

\begin{abstract}
In the aftermath of disasters such as major earthquakes, several roads may be blocked by rubble and the population tends to search refugee in certain gathering points of the city. Road network accessibility becomes an important issue for logistic operations, specially on the first days after the quake, when the relief distribution is crucial for survival. This study focused on the Road Emergency Rehabilitation Problem, divided into the Road Network Accessibility Problem (RNAP) and the Worktroops Scheduling Problem (WSP). The first one consists in finding traversable paths for relief teams to reach the population, and the later generates a repairing schedule to improve access to refugee areas. The contributions of this study are two-fold: we present the process of transcribing satellite imagery data into graphs, and mathematical formulations for the RNAP and WSP, along with heuristics to solve the WSP. The proposed methods are able to handle large-scale graphs in an acceptable running time for real scenarios. They are tested on simulated instances and on the graph of Port-au-Prince, with more than 10,000 vertices and edges. The Port-au-Prince graph was generated from satellite images obtained by the International Charter "Space and Major Disasters" a few hours after the 2010 earthquake.
\end{abstract}

This work is part of the OLIC (Optimisation de la Logistique d'Intervention pour les Catastrophes majeures) project, funded by the Conseil Supérieur de la Formation et de la Recherche Stratégiques (CSFRS), France, and coordinated by Andréa Cynthia Santos.

$凶$ Andréa Cynthia Santos andrea.duhamel@utt.fr

1 Departamento de Engenharia de Produção, Universidade Federal de Sergipe, Av. Marechal Rondon S/N, Jardim Rosa Elze, 49100-000 São Cristóvão, Brazil

2 ICD-LOSI, UMR CNRS 6281, Université de Technologie de Troyes, 12 rue Marie Curie, CS 42060, 10004 Troyes Cedex, France

3 SERTIT, Parc d'Innovation, Bld Sébastien Brant, CS 10413, 67412 Illkirch-Graffenstaden, France 
Keywords Emergency logistics · Post-disaster relief · Network accessibility • Humanitarian logistics · Multi-flow formulations · Heuristics

Mathematics Subject Classification 90B06 Transportation, logistics - 90B10

Network models, deterministic · 90B90 Case-oriented studies · 90C11 Mixed integer programming · 90C90 Applications of mathematical programming

\section{Introduction}

On January 12, 2010, 21:53 UTC (16:53 local time), a 7.0 magnitude earthquake struck Haiti capital Port-au-Prince, followed by several aftershocks with magnitudes over six, causing major damages and casualties as shown in Fig. 1. In the aftermath of major disasters like this one, the population survivability rate is directly related to the delivery of supplies (Duhamel et al. 2014). The $72 \mathrm{~h}$ that follows the quake can be considered the emergency rescue period, after which the chance of survival of the population may decrease dramatically (Feng and Wang 2003). Although resources like helicopters may also be deployed, the majority of the population usually receives assistance and supplies from road relief teams.

In such situations, the population tends to concentrate in gathering points of the city, and it becomes crucial to find routes that allow relief teams to reach the largest part of the population in a minimum time. Thus, road network accessibility becomes an important issue, since a large amount of the city structures might be strongly affected, with rubble blocking several roads. To improve accessibility, Work-troops (WT) composed of bulldozers, excavators, and dump trucks, are deployed to clean and repair the roads.

In this context emerged the project Optimization of Logistics Interventions for major Catastrophes (OLIC), a collaboration between the Industrial Systems Optimiza-

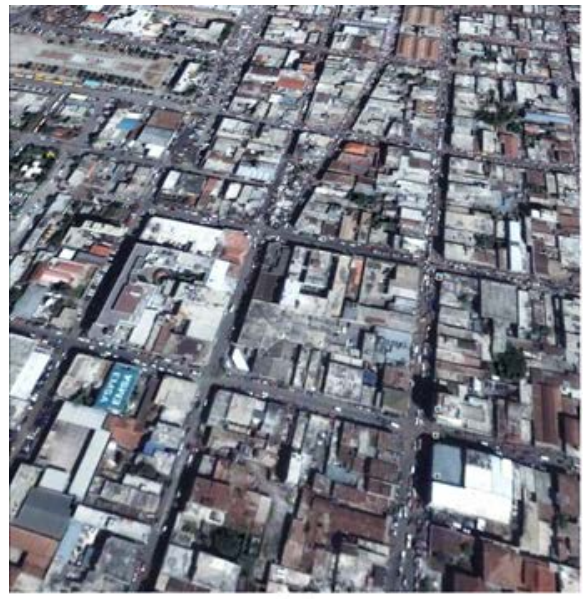

(a)

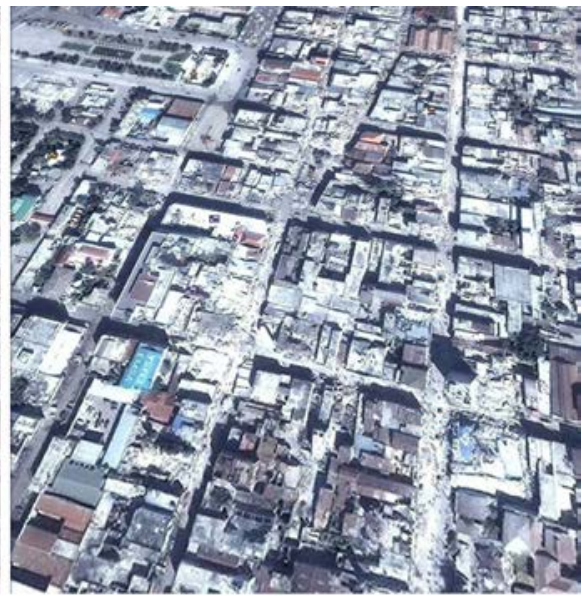

(b)

Fig. 1 One sector of Port-au-Prince 1 day before (a) and after (b) the 2010 earthquake (Source: Google Earth 2016) 
tion Laboratory of Troyes University of Technology (LOSI-UTT), and the Regional Service of Image Processing and Teledetection (SERTIT). The cooperation intends to develop a process of using satellite data from real disasters, collected by the International Charter "Space and Major Disasters" (ICSMD) (2016) and transcribed into graphs by SERTIT, as input for the algorithms proposed by the LOSI-UTT. The idea is to produce solutions in a reasonable time, along with recommendations and insights for future crisis situations.

Differently of other works on similar problems, this research presents tools specially developed to deal with a large amount of data, corresponding to a detailed representation of a whole city affected by a major earthquake. This level of detail is necessary to indicate precisely the routes to be used by relief teams and WT to reach their destinations inside the city. It allows us to evaluate the situation as a whole, obtaining globally better solutions than separating it in regions and handling them apart for each other.

This paper addresses the road accessibility of the population immediately after a major earthquake, defined here as the Road Emergency Rehabilitation Problem (RERP). The RERP is divided into the following problems: the Road Network Accessibility Problem (RNAP) and the Work-troops Scheduling Problem (WSP). The first one consists in determining shortest paths (SP) to areas where the population tends to gather after earthquakes, together with a computation of the time in order to make these areas reachable in the road network immediately after the quake. The WSP defines the order in which blocked edges should be repaired by WT to improve the access to the population gathering areas as fast as possible. We propose mathematical models and algorithms for both problems, which are applied to the data of the Haiti 2010 earthquake. The data was obtained from satellite images hours after the disaster and processed together with an already existing database to estimate the network parameters necessary to solve the problems.

The remaining of the paper is organized as follows: related works are summarized in Sect. 2. Sections 3 and 4 introduce a formal definition of the problems with mathematical models and describe the proposed heuristics for the WSP. Section 5 shows the process of obtaining the data from satellite images and processing it into graphs, and Sect. 6 shows the results of the application of the model and heuristics in simulated and real scenarios. Section 7 presents the concluding remarks and suggestions for future works.

\section{Literature review}

Humanitarian logistics and emergency management are topics involving different areas, requirements, and skills, as shown in the reviews presented by Anaya-Arenas et al. (2014), Caunhye et al. (2012) and Diaz et al. (2013). There are some works dedicated to the road network rehabilitation after disasters, considering emergency and long-term road network repairing, and even static and dynamic cases.

Regarding the long-term road network rehabilitation after earthquakes, the authors in $\mathrm{Hu}$ et al. (2012) analyze the damages after the 8.0 magnitude quake that struck Wenchuan County, China, in 2008. They discuss problems resulting of different damage modes on traffic structures in the road restoration. A fuzzy multi-objective bi-level 
model for road network reconstruction after an earthquake is proposed in Chen and Tzeng (1999). The upper level of the model generates a WT schedule that minimizes total reconstruction time, WT workload differences and total travel time in the road network during reconstruction, weighted by the traffic volume. The lower level is continuously solved by the diagonalization method to assign traffic directions accordingly to the network status. They suppose that each damaged node is entirely repaired by the same WT, and propose a genetic algorithm to allocate them.

Two static versions of emergency road rehabilitation problems, where the order in which edges are repaired is not considered, are presented in Duque and Sorensen (2011) and Feng and Wang (2003). Duque and Sorensen (2011) present a max-flow based model to minimize the weighted sum of the SP to each destination. A Greedy Randomized Adaptive Search Procedure (GRASP) combined with Variable Neighborhood Descent (VND) is proposed based on an efficient calculation of the distances reduction to destinations obtained by repairing each edge. A multi-objective mathematical model that simultaneously maximizes accessibility and the number of lives saved, while minimizes the risks involved in the operation is presented in Feng and Wang (2003). An enumeration of possible repaired road sets for each WT is made over the map of Nantou County, Taiwan, and alternatives are evaluated by a compromise among the three criteria.

Two dynamic versions of the emergency road rehabilitation problems are proposed in Aksu and Ozdamar (2014) and Fiondella (2013). Two mathematical models are introduced by Aksu and Ozdamar (2014) to generate a restoration schedule and the WT distribution among origins. The model uses predetermined SP, and is applied to two regions of Turkey. In Fiondella (2013), a network vulnerability measure based on SP and road risks is introduced, together with a risk mitigation algorithm. The algorithm elaborates a road repair prioritization per time period and is applied to two American cities.

A dynamic representation of the problem using a time-space network is presented in Yan and Shih (2007). In this representation, each node corresponds to an origin, intersection or damaged area at a certain time. A heuristic based on adding damaged points sequentially is presented together with a scenario analysis varying the number of WT. The same network representation is used in Yan et al. $(2012,2014)$ and Yan and Shih (2009). The highway restoration presented by Yan et al. (2014) considers the rescheduling of WT due to changes in the repair demands. A hybrid global search based on the ant colony metaheuristic is proposed. In Yan et al. (2012), the objective is to minimize the operational cost of bringing several materials used to repair the road network to the places where they are needed. A mathematical model is presented and solved by the decomposition and variable fixing techniques. A model to minimize the time to both restore all damaged roads and distribute all the relief is presented in Yan and Shih (2009). The model is solved by dividing the problem into sub-problems, in which repair points are added to the models in different orders, and handling them with CPLEX solver.

The works presented in this section have been applied to realistic scenarios in different cities throughout the world. However, there is a lack of research to handle an entire network in its details after large-scale disasters as Port-au-Prince 2010 earth- 
quake. Even the map of Port-au-Prince after the 2008 hurricane used in Duque and Sorensen (2011) was a simplified version of the road network, with a few hundred nodes and roads, as most of the works presented in this section. We model a more complex problem closer to large-scale ones found in practice, and develop simple and efficient algorithms able to provide solutions in a reasonable amount of time. The goal is to provide solutions and insights for solving the RERP in real contexts. Furthermore, as last but not least contribution, we show the data treatment process to obtain the graphs and parameters.

\section{Problem statements and formulations}

The RERP was divided into two distinct problems, RNAP and WSP, defined in the following subsections along with their mathematical formulations. The first problem corresponds to the computation of access routes in the original state of the damaged network. Besides, an estimation of the minimum number of time periods to make the population accessible is computed. The second one consists of building a WT scheduling per time period, to improve dynamically the access to the population gathering areas as fast as possible along a time horizon.

A connected graph $G=(V, E)$ is defined for both problems, where $V$ is the set of $n$ vertices and $E$ the set of $m$ undirected edges corresponding to road segments of the region in study. The direction of the edges was not considered, since in emergency logistics and operations inside cities, the direction of the roads may be ignored, providing that the vehicles have to run in reduced speed given the streets condition. Let $O \subset V$ be the subset of origins, i.e., entry points of the network from where WT and relief teams depart, and $D \subset V$ the subset of destinations corresponding to population gathering zones $(O \cap D=\oslash)$. Let $Q$ be the total number of available WT. Each origin has a number $q_{i} \in \mathbb{N}^{*}$ of available WT $\left(\sum_{i \in O} q_{i}=Q\right)$, and each destination has a population $p_{i} \in \mathbb{N}^{*}$.

Each edge $[i, j]$, with $i<j$, is associated with a set of three parameters described as follows. The distance $d_{i j} \in \mathbb{R}_{+}^{*}$ is the length of the edge. The repair effort $r_{i j} \in \mathbb{N}$ represents the amount of working periods necessary for one WT to repair that edge. If $r_{i j}=4$ for example, the edge is repaired after one WT spends four time periods working on it, two WT spend two time periods each, or in one time period by four WT simultaneously, if the road width allows it. We define $B \subset E$ the subset of blocked edges, i.e., non-trafficable roads that require repair, for which $r_{i j}>0\left(r_{i j}=0\right.$ for all $[i, j] \notin B$ ). Due to physical limitations imposed by the road width, the maximum number of WT that can work simultaneously at each extremity of a road is given by an upper bound $u_{i j} \in \mathbb{N}^{*}$. Moreover, $T$ is the number of time periods considered in the models.

The models presented in the following subsections make use of $G^{\prime}$, a digraph conversion of $G$ obtained by replacing each edge $[i, j] \in E$ by $\operatorname{arcs}(i, j)$ and $(j, i)$, generating the set of $\operatorname{arcs} A$. The digraph also contains an artificial root vertex 0 connected to all origins by $\operatorname{arcs}(0, i)$, with $d_{0 i}=r_{0 i}=0, \forall i \in O$. Thus, $G^{\prime}=$ $\left(V^{\prime}, A^{\prime}\right)$ is composed of the vertex set $V^{\prime}=V \cup\{0\}$ and the $\operatorname{arc} \operatorname{set} A^{\prime}=A \cup\{(0, i) \mid i \in$ $O\}$. 


\subsection{The road network accessibility problem}

A problem that emerges immediately after an earthquake is the calculation of unblocked SP from origins to destinations. The RNAP is to find such paths, along with the fastest repairable paths to destinations that are unreachable, evaluating the minimum time to make them traversable. It is important to mention that destinations do not have to be reached from the same origin, and that in the RNAP, the limitation on the number of WT is not considered.

We propose a multi-flow formulation to determine the initial accessibility of the damaged network and estimate the time to make each destination reachable. Its main idea is to send one flow unit from an origin to each destination. Traversing costs are set as $t_{i j}=\left\lceil\frac{r_{i j}}{u_{i j}}\right\rceil K+d_{i j}$ for all $\operatorname{arcs}(i, j) \in A^{\prime}$, where $K$ is a weight to give priority to the repair time of blocked arcs. It must be selected to ensure that $\left\lceil\frac{r_{i j}}{u_{i j}}\right\rceil K>d_{i j}$ for all arcs.

We took $K=\frac{d_{\max }}{\left\lceil r_{\min } / u_{\max }\right\rceil}+1$, where $d_{\max }$ is the maximum arc length, $r_{\min }$ the minimum repair effort, and $u_{\max }$ the maximum of $u_{i j}$. Since we are interested only in cases where there are several blocked roads, we assume $r_{\min }>0$. Variables $f_{i j}$ represent the flow on arc $(i, j), x_{i j}$ denote if there is a flow on arc $(i, j)$ or not, and $y_{i}$ estimate the time period from which vertex $i$ can be reached.

$$
\begin{aligned}
& \min \quad z=\sum_{i \in D} p_{i} y_{i} \quad \text { s.t. } \\
& \sum_{(0, i) \in A^{\prime}: i \in O} f_{0 i}=|D| \\
& \sum_{i:(i, j) \in A^{\prime}} f_{i j}-\sum_{i:(j, i) \in A^{\prime}} f_{j i}=0 \quad \forall j \in V^{\prime} \backslash D \\
& \sum_{i:(i, j) \in A^{\prime}} f_{i j}-\sum_{i:(j, i) \in A^{\prime}} f_{j i}=1 \quad \forall j \in D \\
& y_{i}-y_{j}+\left(M+t_{i j}\right) x_{i j} \leq M \quad \forall(i, j) \in A^{\prime} \\
& x_{i j} \geq \frac{f_{i j}}{|D|} \quad \forall(i, j) \in A^{\prime} \\
& x_{i j} \leq f_{i j} \\
& y_{i} \geq 0 \\
& \forall(i, j) \in A^{\prime} \\
& \forall i \in V^{\prime} \\
& f_{i j} \geq 0 \\
& \forall(i, j) \in A^{\prime} \\
& x_{i j} \in\{0,1\}
\end{aligned}
$$

The objective (1) is to reach the maximum number of population in a minimum time. Equations (2)-(4) represent the flow conservation constraints and inequalities (5) calculate the times each destination becomes reachable from an origin. Constraints (6) and (7) link variables $f_{i j}$ to $x_{i j}$. Variables are defined from (8) to (10). The formulation has $O\left(\left|A^{\prime}\right|\right)$ variables and constraints. 
Our graph being symmetric, when two nodes $i$ and $j$ are connected by two opposite arcs, the difference $y_{i}-y_{j}$ cannot exceed $t_{i j}$. Indeed, assume without loss of generality that $y_{i}>y_{j}$ : if $y_{i}-y_{j}>t_{i j}$, we could improve the SP to $i$ by taking the SP to $j$ and adding $(j, i)$. Hence, in constraints (5), $M$ can be selected as the maximum of the $t_{i j}$.

One may note that the RNAP provides a quick evaluation of unblocked SP, i.e., paths to destinations for which $y_{i}<M$, estimating the time to repair blocked SP by the coefficient of $M$ in their values of $y_{i}$ (integer part of the result of $y_{i} / M$ ). This model can be solved in polynomial time by finding SP from the artificial root vertex to the destination points. For this purpose, several efficient algorithms, such as Bellman (1958) and Dijkstra (1959), can be used to solve even instances as large as Port-au-Prince (Sakuraba et al. 2015).

\subsection{The work-troops scheduling problem}

After a network accessibility analysis, the next step is to generate a WT schedule to repair blocked edges in a way that accessibility to destinations is improved as fast as possible. We define this problem as the Work-troops Scheduling Problem (WSP) and propose the mathematical formulation (11)-(28) considering the dynamic aspect of the problem, i.e., the network evolution along time as edges are repaired. SP to destinations and WT access to blocked edges are computed at each time period.

The model has two main components per time period: a flow from an origin to each destination through its SP and a WT flow from the origins to an extremity of a blocked edge. Let $B^{\prime} \subset A$ be the subset of all arcs obtained by replacing blocked edges $[i, j] \in B$ by blocked $\operatorname{arcs}(i, j)$ and $(j, i)$. We consider that a WT flow cannot pass through an arc $(i, j) \in B^{\prime}$, and its value of $d_{i j}$ is set to a large integer number $M$ when determining the SP flow.

The model uses the following variables: at the end of each time period $t$, the SP flow through arc $(i, j)$ is represented by variables $f_{i j}^{t}$, and variables $x_{i j}^{t}=1$ if arc $(i, j)$ is used on some SP, 0 otherwise. Besides, variables $z_{i j}^{t}=1$ if edge $[i, j]$ is available and 0 otherwise $\left(\sum_{[i, j] \in B} z_{i j}^{0}=0\right)$, and variables $s_{i}^{t}$ state the length of the SP from the root vertex 0 to a vertex $i$. During each time period $t$, the WT flow on arc $(i, j)$ is determined by variables $y_{i j}^{t}$, while variables $w_{i j}^{t}$ represent the number of WT entering arc $(i, j)$ to repair it. Note that WT can repair edges from both extremities, with $w_{i j}^{t}>0$ and $w_{j i}^{t}>0$ for an edge $[i, j] \in E$ and $1 \leq t \leq T$.

$$
\begin{aligned}
& \min Z=\sum_{i \in D} \sum_{t=1}^{T} p_{i} s_{i}^{t} \quad \text { s.t. } \\
& \sum_{i:(i, j) \in A^{\prime}} f_{i j}^{t}-\sum_{i:(j, i) \in A^{\prime}} f_{j i}^{t}=\left\{\begin{array}{ll}
|D|, & \text { if } i=0 \\
0, & \forall j \in V \backslash D, \\
1, & \forall j \in D
\end{array} \quad t=1, \ldots, T\right. \\
& x_{i j}^{t} \geq \frac{f_{i j}^{t}}{|D|} \\
& x_{i j}^{t} \leq f_{i j}^{t} \\
& \sum_{j:(i, j) \in A} y_{i j}^{t}+\sum_{k:(i, k) \in B^{\prime}} w_{i k}^{t} \leq q_{i} \\
& \begin{array}{r}
\forall(i, j) \in A^{\prime}, t=1, \ldots, T \\
\forall(i, j) \in A^{\prime}, t=1, \ldots, T \\
\forall i \in O, t=1, \ldots, T
\end{array}
\end{aligned}
$$




$$
\begin{aligned}
& \sum_{i:(i, j) \in A} y_{i j}^{t}-\sum_{i:(j, i) \in A} y_{j i}^{t}=\sum_{k:(j, k) \in B^{\prime}} w_{j k}^{t} \\
& \sum_{[i, j] \in B} z_{i j}^{T}=|B| \\
& r_{i j} z_{i j}^{t} \leq \sum_{t^{\prime}=1}^{t}\left(w_{i j}^{t^{\prime}}+w_{j i}^{t^{\prime}}\right) \\
& y_{i j}^{t}+y_{j i}^{t} \leq \sum_{i \in O} q_{i} z_{i j}^{t-1} \\
& w_{i j}^{t} \leq u_{i j} \\
& s_{j}^{t} \geq M\left(x_{i j}^{t}-z_{i j}^{t}\right) \\
& s_{i}^{t}-s_{j}^{t}+\left(M+d_{i j}\right) x_{i j}^{t} \leq M \\
& f_{i j}^{t} \geq 0 \\
& s_{i}^{t} \geq 0 \\
& w_{i j}^{t} \geq 0 \\
& x_{i j}^{t} \in\{0,1\} \\
& y_{i j}^{t} \geq 0 \\
& z_{i j}^{t} \in\{0,1\}
\end{aligned}
$$

$$
\begin{aligned}
\forall j \in V \backslash O, t & =1, \ldots, T \\
\forall[i, j] \in E, t & =1, \ldots, T \\
\forall[i, j] \in E, t & =1, \ldots, T \\
\forall(i, j) \in A, t & =1, \ldots, T \\
\forall(i, j) \in A^{\prime}, t & =1, \ldots, T \\
\forall i, j \in V \mid(i, j) \in A^{\prime}, t & =1, \ldots, T \\
\forall(i, j) \in A^{\prime}, t & =1, \ldots, T \\
\forall i \in V^{\prime}, t & =1, \ldots, T \\
\forall(i, j) \in A, t & =1, \ldots, T \\
\forall(i, j) \in A^{\prime}, t & =1, \ldots, T \\
\forall(i, j) \in A, t & =1, \ldots, T \\
\forall[i, j] \in E, t & =1, \ldots, T .
\end{aligned}
$$

The objective function (11) is the weighted sum of the SP distances to each destination over all time periods. Equations (12) ensure that there is a unitary flow from the root vertex 0 to each destination, while inequalities (13) and (14) link variables $f_{i j}^{t}$ to $x_{i j}^{t}$. Constraints (15) and (16) represent the WT flow, limited to the number $q_{i}$ of WT available at each origin, allocating them to blocked edges. Equation (17) guarantees that all edges initially blocked are fully repaired by the last time period, while inequalities (18) set the edges availability at each time period according to the number of working periods spent on repairing it until the present time period. Constraints (19) make WT use only available edges to reach blocked edges. Inequalities (20) limit the number of WT on each extremity of a blocked edge according to the road physical limitation. Constraints (21) make the distance of the SP to a vertex equal to $M$ if all the paths to that vertex are blocked. In this model, we assume $M$ as the maximum SP distance between any two vertices of the graph. The distances of the SP to each destination from its nearest origin are calculated in constraints (22), by accumulating the distances of the SP from this origin to each intermediate vertex in the path. Variables are defined from (23) to (28). This formulation contains $O\left(\left|A^{\prime}\right| \times T\right)$ variables and constraints.

\section{Proposed heuristics}

We proposed three heuristics for the WSP, able to produce solutions for large-scale graphs as the Port-au-Prince one generated in this study. We focus on simple heuristics due to time constraints that exist when producing solutions for emergency situations. Moreover, many runs are usually performed by the user due to the chaotic situation and 
several unexpected events, such as earthquake aftershocks, vehicle failures, buildings, which collapse during the relief operations, etc. In spite of their simplicity, the proposed heuristics are able to produce high quality results. The heuristics are presented with their pseudo-codes in the following subsections. All of them require SP calculations, which were performed using a binary heap implemetation of Dijkstra's algorithm [which runs in $O(m \log n)$ Cormen et al. (2009)], and output a solution composed by a WT schedule and the value of the objective function (OF).

\subsection{Savings heuristic}

The first heuristic, henceforth referred as Savings Heuristic ( $\mathrm{SH}$ ), is based on the calculation of edge savings similar to the ones defined in Duque and Sorensen (2011). SH has the following stages: initially, WT are pre-allocated to edges in blocked SP to destinations that can be unblocked already in the first time period. Then, remaining blocked edges are ranked in non-increasing order of their savings. The savings of an edge $[i, j] \in B$ are the reduction in the weighted sum of SP to destinations $\left(\sum_{i \in D} p_{i} s_{i}^{t}\right)$ in the current period $t$ once $[i, j]$ is completely repaired. The ranking is used as a priority list to allocate WT in each consecutive time period. Savings are evaluated, and the ranking is updated every time an edge is completely repaired.

The framework of SH is shown in Algorithm 1. For each origin, Dijkstra's algorithm (1959) is used to find SP to all vertices using $r_{i j}$ values, determining which vertices are accessible to each WT (line 3). A list is made with edges in SP to unreachable destinations containing only one blocked edge, meaning that it may be possible to repair the SP in one time period (lines 4-8). Savings are computed for all blocked edges and ranked in non-increasing order (lines 10-13). Lines 14-16 make a preallocation of WT to the edges in the list if they can be repaired immediately, i.e., its width allows enough WT to repair it in one time period. Then, for each time period, available WT are allocated to the first workable edge in the ranking, i.e., the first edge they can reach that supports more WT given the road width limitation (lines 18-20). If some blocked edge is completely repaired, the savings are updated and edges still blocked are ranked again (lines 21-25). Besides, for each edge completely repaired, $\mathrm{SP}$ values from origins to all vertices are updated (lines 26-28). To evaluate changes in SP distances from each origin $o \in O$ to a vertex $v \in V$ efficiently every time, an edge $[i, j]$ is completely repaired, we calculated SP from $i$ and $j$ to all other vertices. The distance from $o$ to $v$ is updated if the original SP is longer than the sum of three terms: the $\mathrm{SP}$ from $o$ to $i$ (or $j$ ), $d_{i j}$, and the $\mathrm{SP}$ from $j$ (or $i$ ) to $v$.

\subsection{Ranking heuristic}

The Ranking Heuristic (RH) allocates WT according to a blocked edges ranking based on counting the number of times they appear in SP from origins to destinations. SP from each pair origin-destination are found, and the number of times each edge appears in the SP defines their rank value. Edges are ranked in non-increasing order of their rank. WT are sent to the first workable edge from the top of the ranking after a pre-allocation like the one in $\mathrm{SH}$. 


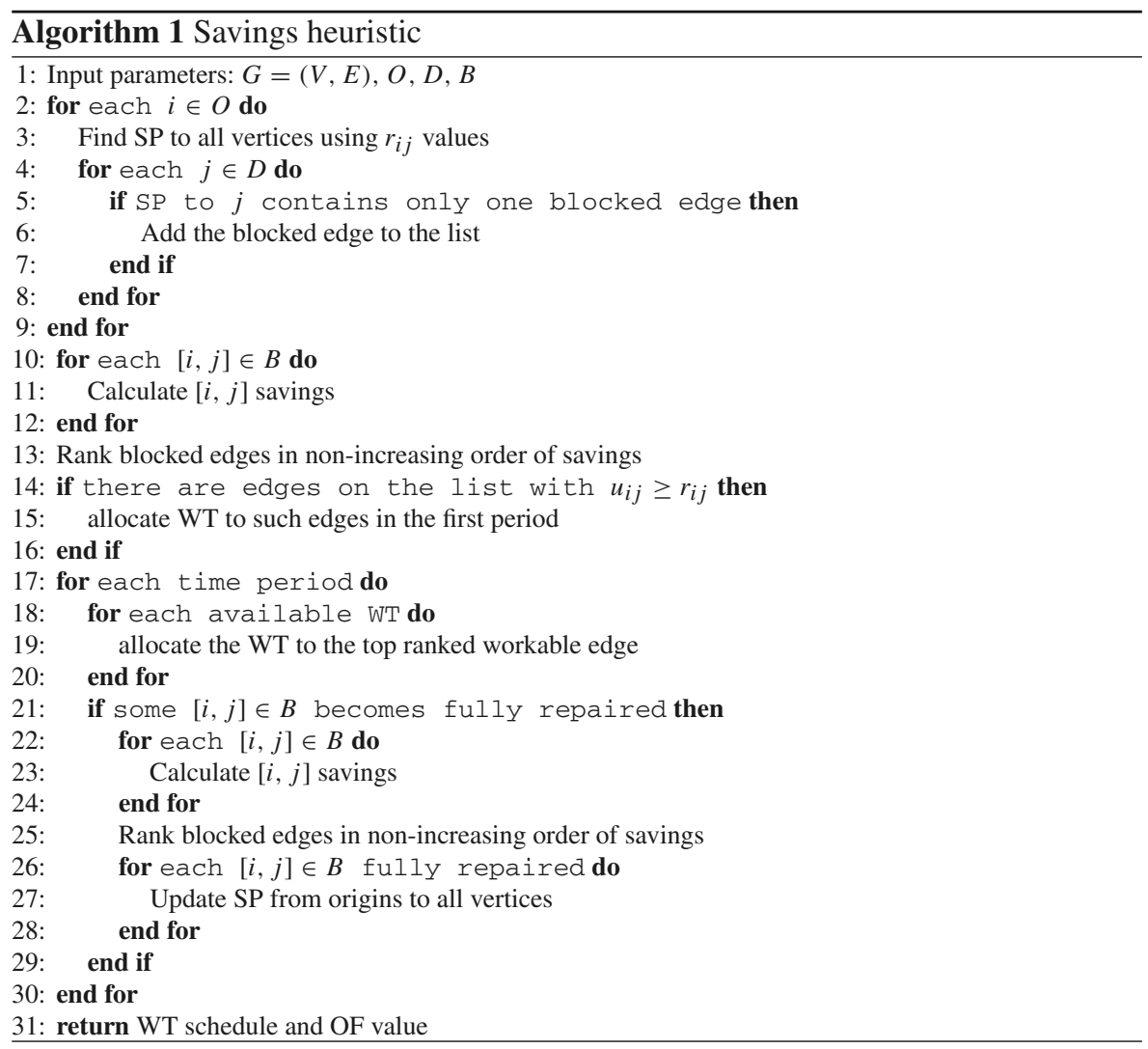

Algorithm 2 presents a pseudo-code for RH. The first steps of RH (lines 2-10) are similar to $\mathrm{SH}$, with the additional counting of the number of times blocked edges appear in SP to destinations (line 5). Edges are ranked in non-increasing order of the number of times they appear in SP (line 11). Ties are broken by the smallest value of $r_{i j}$, with edges that can be quickly repaired being handled first. Then, the same WT pre-allocation and allocation to workable blocked edges in the ranking order as $\mathrm{SH}$ is made per time period from (lines 12 and 23). SP are updated whenever an edge is completely repaired (line 20), without rearranging the edge ranking.

\subsection{Lexicographic classification heuristic}

The Lexicographic Classification Heuristic ( $\mathrm{LCH}$ ) works with two candidate sets, S1 and $\mathrm{S} 2$, both containing blocked edges in paths to destinations. Each set contains one edge list per destination, with two criteria classifying edges into one of the sets: the time necessary to repair the path and the path distance. S1 has edges belonging to the fastest repairable path to each destination, i.e., edges that can be repaired in a smaller amount of time periods to create an unblocked path to a destination. These edges are determined by applying the model for the RNAP (Sect. 3.1), with the list 


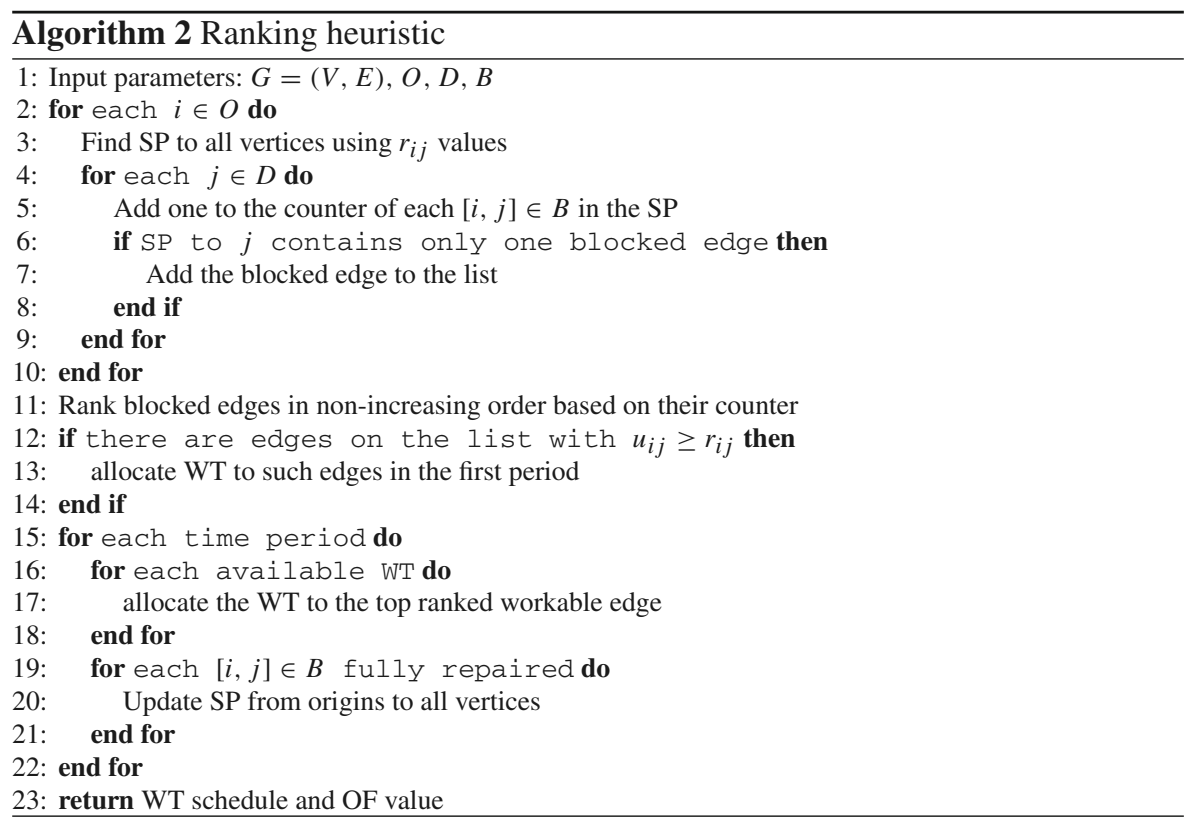

being empty if the destination is reachable from the beginning. S2 keeps edges in the SP to each destination considering only edge lengths $d_{i j}$, regardless if they are blocked or not. Edges on both sets are kept arranged by destination, in the same order they appear in their path, since only the first edge in a blocked path is reachable to WT.

$\mathrm{LCH}$ repairs edges from the sets according to the network state in the current time period. If there are still unreachable destinations, WT are allocated to edges from S1, repairing a path to these destinations as fast as possible. Otherwise, WT are sent to repair edges in S2, unblocking the SP to each destination. Sets are scanned starting from the edges in the path to the most populated destination. WT are allocated to the first workable edge until its upper bound $u_{i j}$ is reached. Then, edges on the paths to other destinations are scanned likewise, in non-increasing order of their population. Edges on paths to different destinations are repaired simultaneously by WT working in parallel. Once all edges in SP to all destinations are repaired, WT are randomly allocated to workable edges for the following time periods.

The general structure of LCH is presented in Algorithm 3. The first set L1 is generated based on the solution of the RNAP (lines 2-4). Dijkstra's algorithm is applied to find fastest repairable paths from each origin to all vertices, evaluating also their reachability for WT allocation. S1 is built with the blocked edges in the paths to each destination with the smallest repairing time among origins. Then, Dijkstra's algorithm is applied again to determine SP to destinations based only on edge lengths $\left(d_{i j}\right)$, and blocked edges in the SP are kept in S2 (line 5).

Once sets are built, the heuristic allocates WT in chronological order, starting from the first time period (lines 6-21). For each time period, each WT is allocated as follows: 


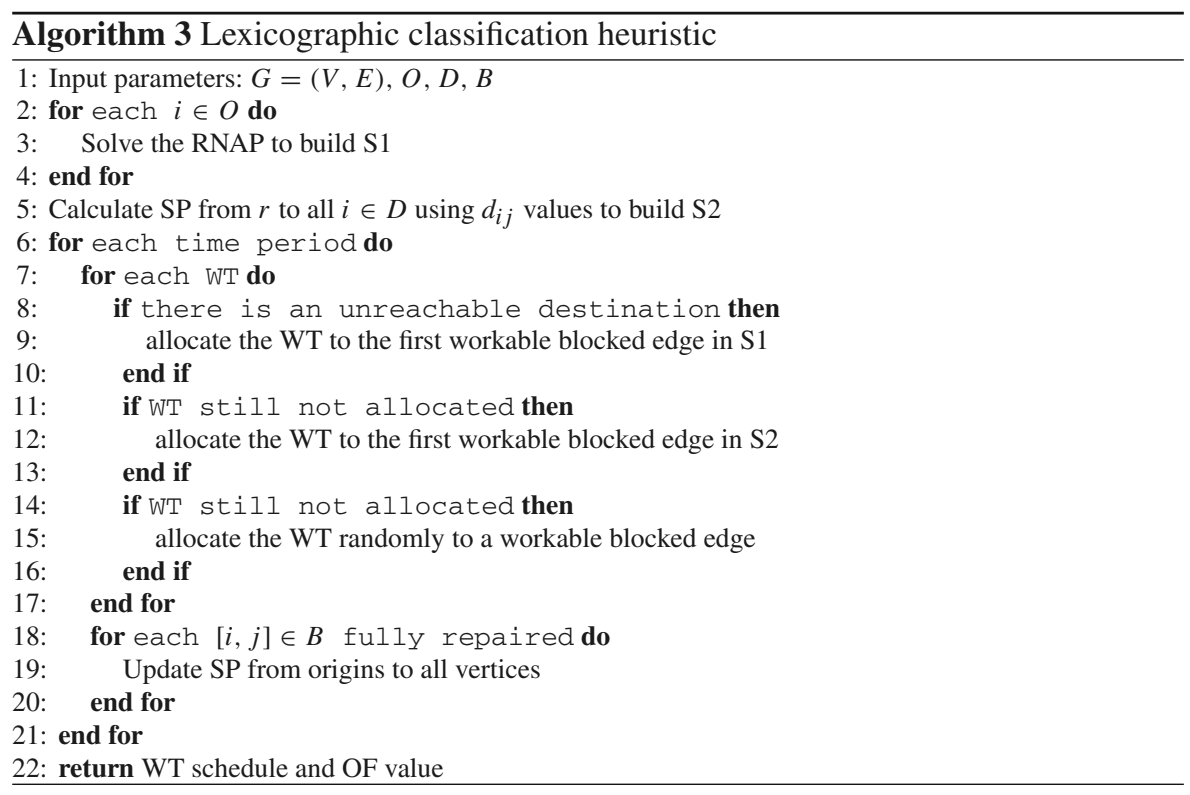

lists for unreachable destinations in S1 are scanned, and the WT is allocated to the first workable edge found (lines 8-10). If all destinations are reachable or the WT could not be allocated to any blocked edge in S1, S2 is scanned likewise to allocated it from lines 11-13. In case the WT remains unallocated, allocate it randomly to a workable blocked edge (lines 14-16). After allocating all WT for a time period, the SP are updated with the completely repaired edges (lines 18-20).

\section{Data processing}

This section describes the 2010 earthquake data processing into a format that allows the solution of the RERP. This process involves gathering data obtained from satellite images after the quake, coupled with some network information previously digitalized, providing a graph for Port-au-Prince with real data associated with the population gathering points, roads condition, etc. The general framework of the event is described together with the process of obtaining the satellite image, followed by the transcription of the data into a graph representing the road network condition after the quake.

Two hours after the 2010 earthquake in Port-au-Prince, the ICSMD was first triggered by the French Civil Security. In parallel, Global Monitoring for Environment and Security's (GMES) SAFER was triggered. The GMES's SAFER was a European Union-funded project responsible for the development of the pre-operational GMES's emergency management service. It contributed to the Charter by producing valuable additional damage maps. Nowadays, the service has evolved and is named Copernicus Emergency Management Service (2016). 
The first very high-resolution image (pixel size: $0.41 \mathrm{~m}$ ) was acquired by the optical satellite GeoEye-1 17 hours after the earthquake and made available to download on January 14, 07:31 UTC. The first damage evaluation over the city of Port-au-Prince was published by SERTIT on January 14, 15:08 UTC, a little more than $41 \mathrm{~h}$ after the quake. During the two following weeks, hundreds of satellite images were acquired, and numerous maps were produced by several entities.

On January 14, 2010, the center for satellite-based crisis information of the German Aero-space Center (DLR/ZKI) published a set of pre-disaster maps showing an extensive road network over the urban area of Port-au-Prince. The set of roads was provided by the United Nation's Cartographic Section and completed by DLR/ZKI by digitalization over satellite imagery. However, a more extensive and topologicallyconsistent road network was needed to provide all parameters required by the models presented in Sect. 3.

A road network previously produced in the framework of the KAL-Haiti project (Giros et al. 2012) was used to obtain a graph representation of the network. It has been digitalized over aerial images (pixel size: $15 \mathrm{~cm}$ ) acquired on January 2010 and provided by Google Inc. In the digitalization, each road section could have several nodes. Thus, the data treatment described on the next paragraphs was performed in this research, rewriting the graph by considering each section as a segment with only one origin and end point, as shown in Fig. 2a.

The attributes corresponding to each road section were obtained from the images. The section length in meters $\left(d_{i j}\right)$ was measured using the geometry of the layer. A classification of the road types was made by photo-interpretation over the same aerial images used for digitalization also using the geometry. Five categories were generated: main road, secondary road, tertiary road, road only usable by cars (too narrow for a truck), and pedestrian paths. This classification was used to determine the parameter $u_{i j}$ as follows: three for main roads, two for secondary and tertiary roads, and one for the remaining ones. The roads condition, derived from photo-interpretation of satellite imagery, were categorized in four levels used to estimate the repair $r_{i j}$. The values of $r_{i j}$ were defined as zero for trafficable roads, one for lightly blocked ones, two for intermediate blockage, and three for severe blockage. An example of the different blockage levels is shown in Fig. 2 b.

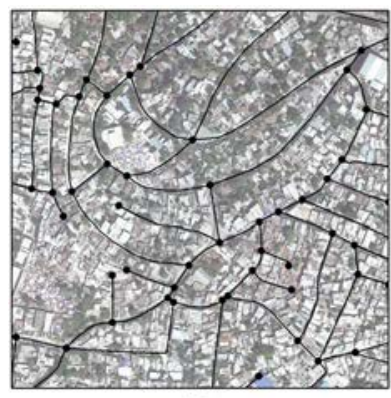

(a)

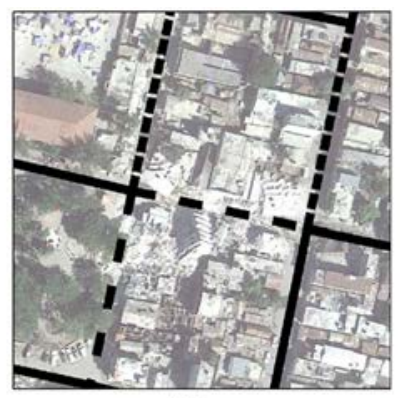

(b)

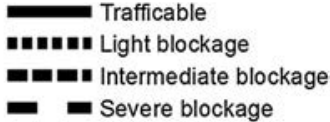

Fig. 2 Examples of graph representation of the road network (a) and road-condition categories (b) 


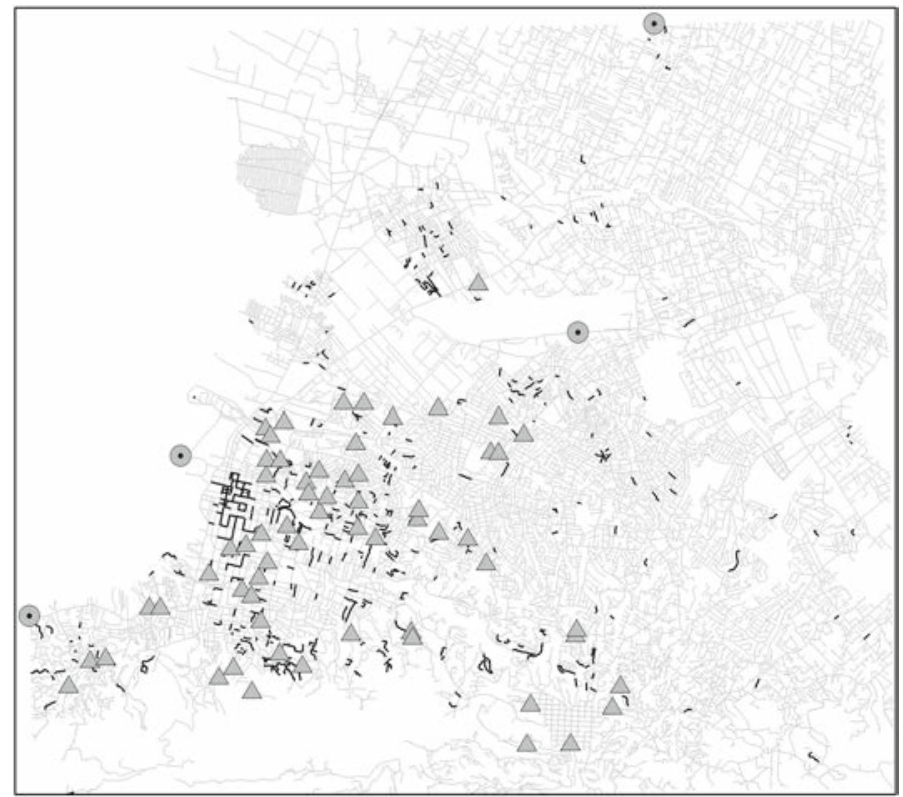

Fig. 3 Port-au-Prince road network after the 2010 earthquake. Circles, triangles, and dark edges represent origins, destinations, and blocked roads, respectively

Two attributes were obtained for each node of the network. The first is the node type, three-folded into: regular node; entry point by road (main access road), sea (port), or air (airport); and spontaneous gathering zone or camp. The two last types of nodes correspond, respectively, to our models set of origin and destination points $(O$ and $D)$. The second attribute concerns the nodes corresponding to the gathering zones, and contains the population $p_{i}$ on that node, computed based on the amount of tents visible in the satellite images.

The data model was generated using two structures, one for nodes and one for edges. The structure for a node contains the following parameters: ID, XUTM, YUTM, node type, and population, which stand, respectively, for the node ID, its latitude and longitude in the Universal Transverse Mercator coordinate system (UTM), the node type as explained before, and the population for gathering zones. The edge's structure contains its ID, the ID's of the start and end nodes, and the three edge attributes enumerated before.

The nodes and edges were scanned one-by-one, and their attributes were transcribed into two ASCII-files representing the graph, where each line corresponds to a node or edge. The resulting graph used in this work contains 16,657 nodes and 19,558 edges, see Fig. 3.

Besides the road network definitions, it is important to define the time period in the context of real applications. In this work, we define one time period as the amount of time required for one WT to clean a certain area of debris, calculated from the satellite images. Since the volume of the debris is proportional to its area, this roadcondition categorization is consistent with the definition presented in Yan and Shih (2009). 


\section{Computational experiments}

Experiments for the WSP were addressed to check the performance of the proposed heuristics. Besides comparisons in terms of computational time and objective function value, we introduce a measure of accessibility to better compare the quality of the solutions found by the heuristics. The accessibility of a solution is defined as the first time period from which all destinations become reachable. Along with its obvious importance in real scenarios, this measure represents a good method to compare the solutions quality, since objective function values may have different orders of magnitude according to the presence or not of a big number $M$.

A set of 80 simulated instances and the graph of Port-au-Prince obtained hours after the earthquake were used in the experiments. We assumed $q_{i}=2, \forall i \in O$, for all instances. Tests were performed on an Intel Core i7-4600M CPU with $2.90 \mathrm{GHz}$ and 16 GB of RAM. The heuristics were implemented in C ANSI, and CPLEX 12.6 was used to solve the simulated instances to optimality.

\subsection{Results for simulated instances}

The simulated instances were randomly generated over a graph with $n=10, m=$ $20,|O|=2$, and $|D|=3$. Ten different blocked edge configurations were generated for each value of $|B|=\{5,10,15,20\}$. For each configuration, two instances were generated with $R=\sum_{[i, j] \in B} r_{i j}=40$ and 45 . The determination of $T$ required preliminary tests to guarantee the feasibility of all instances. $T$ was set to 13 to all simulated instances, and the smallest value for which all of them are feasible. Table 1 summarizes the results obtained by CPLEX within a time limit of $2 \mathrm{~h}$.

Each line in the Table 1 corresponds to a set of ten instances, representing a combination of $R$ and $|B|$ parameters previously defined. The three first columns present the instance set number and the values of $R$ and $|B|$, respectively. The number of instances in each set that exceeded $2 \mathrm{~h}$ is given in column "nb exceeded". The three last columns show, respectively, the average values of: time in seconds $(t(\mathrm{~s}))$; number of nodes visited in the CPLEX B\&B (nb nodes); and accessibility (access.). $90 \%$ of

Table 1 Results obtained by CPLEX on simulated instances

\begin{tabular}{|c|c|c|c|c|c|c|}
\hline Inst.set \# & $R$ & $|B|$ & nb time exceeded & $t(\mathrm{~s})$ & nb nodes & Access. \\
\hline 1 & 40 & 5 & 0 & 12.63 & 9090.5 & 1.5 \\
\hline 2 & & 10 & 1 & 882.92 & $160,058.1$ & 1.4 \\
\hline 3 & & 15 & 1 & 936.65 & $72,434.9$ & 2.1 \\
\hline 4 & & 20 & 0 & 83.65 & $14,348.0$ & 2.8 \\
\hline 5 & 45 & 5 & 0 & 23.30 & $11,980.7$ & 1.7 \\
\hline 6 & & 10 & 2 & 1518.22 & $223,138.5$ & 1.8 \\
\hline 7 & & 15 & 3 & 2277.90 & $108,982.0$ & 2.5 \\
\hline 8 & & 20 & 1 & 818.18 & $39,447.4$ & 3.2 \\
\hline
\end{tabular}


Table 2 Comparison among CPLEX and the proposed heuristics for the simulated instances

\begin{tabular}{|c|c|c|c|c|c|c|c|c|c|c|c|c|}
\hline \multirow[t]{2}{*}{ Inst. set \# } & \multicolumn{3}{|c|}{ CPLEX } & \multicolumn{3}{|l|}{ SH } & \multicolumn{3}{|l|}{ RH } & \multicolumn{3}{|l|}{ LCH } \\
\hline & Avg. & Wst. & Bst. & Avg. & Wst. & Bst. & Avg. & Wst. & Bst. & Avg. & Wst. & Bst. \\
\hline 1 & 1.5 & 4 & 1 & 3.4 & 13 & 1 & 1.5 & 4 & 1 & 1.5 & 4 & 1 \\
\hline 2 & 1.4 & 3 & 1 & 4.6 & 13 & 1 & 1.5 & 3 & 1 & 1.4 & 3 & 1 \\
\hline 3 & 2.1 & 3 & 1 & 6.2 & 13 & 1 & 3.5 & 5 & 1 & 2.2 & 3 & 1 \\
\hline 4 & 2.8 & 4 & 2 & 10.1 & 13 & 2 & 3.6 & 5 & 2 & 2.9 & 4 & 2 \\
\hline 5 & 1.7 & 6 & 1 & 3.4 & 13 & 1 & 1.7 & 6 & 1 & 1.7 & 6 & 1 \\
\hline 6 & 1.8 & 4 & 1 & 5.8 & 13 & 1 & 2.3 & 8 & 1 & 1.8 & 4 & 1 \\
\hline 7 & 2.5 & 4 & 1 & 9.7 & 13 & 1 & 3.8 & 5 & 2 & 2.5 & 4 & 1 \\
\hline 8 & 3.2 & 4 & 2 & 12.0 & 13 & 3 & 3.9 & 5 & 3 & 3.2 & 4 & 2 \\
\hline
\end{tabular}

the instances were solved to optimality, and for the instances which were not solved to optimality, the best solutions found had a deviation of less than $0.5 \%$ from their lower bound. The experiments suggest instances with 10 or 15 blocked edges are somehow difficult to be solved to optimality by CPLEX. A possible explanation is that if only a few edges or the whole graph are blocked, CPLEX is able to take a global decision over the network accessibility. This is not the case when an intermediate number of blocked edges (e.g., 10 or 15) is set, since the combinatorial choices may increase.

The results of the proposed heuristics were compared to the ones obtained by CPLEX. Table 2 presents the accessibility values obtained by CPLEX and by the proposed heuristics. The first column indicates the instance set number. The following columns contain the average (avg.), worst (wst.), and best (bst.) accessibility values found by CPLEX, SH, RH, and $\mathrm{LCH}$, respectively. Values in bold represent the best averages for each instance set.

All heuristics needed less than 0.02 s to solve any of the simulated instances, while CPLEX takes on average $615 \mathrm{~s}$ to find the optimal solution and $820 \mathrm{~s}$ to finish the branch-and-bound tree. We observed that SH and RH could make the whole population accessible in the same time period as CPLEX for 41 and 54 out of 80 instances, respectively. For the remaining instances, $\mathrm{SH}$ obtained an average difference of 4.7 time periods, while for $\mathrm{RH}$ this difference was of 0.6.

The results in Table 2 show that RH and LCH worst case accessibility values are not much different from the ones found by CPLEX, demonstrating their stability. LCH has the best overall performance among the heuristics, with an average accessibility very similar to the solutions obtained by CPLEX. Only one instance of set 3 has a one unit difference on the accessibility compared to CPLEX solutions.

$\mathrm{LCH}$ results are also compared to the ones obtained by CPLEX in terms of their objective function in Table 3. The table shows the number of best-known solutions (nb best.) found for each instance set (i.e., the number of instances for which the heuristic found the same results as CPLEX) and the average relative deviation (diff. \%) to the optimal or best solution found by CPLEX solver for the ten instances. LCH was able 
Table 3 Average results for the ten simulated instances in each set

\begin{tabular}{lll}
\hline Inst.set \# & \multicolumn{2}{c}{ Lexicographic classification heuristic } \\
\cline { 2 - 3 } & nb best. & Diff. \% \\
\hline 1 & 9 & 0.21 \\
2 & 4 & 5.27 \\
3 & 5 & 8.73 \\
4 & 8 & 2.49 \\
5 & 9 & 0.09 \\
6 & 5 & 2.72 \\
7 & 5 & 0.57 \\
8 & 8 & 1.87 \\
\hline
\end{tabular}

to obtain the optimal solution for approximately two-thirds of the instances (53 out of 80 ), and for $85 \%$ of the instances with $|B|=5$ or 20 . If we consider only instances for which LCH solutions are different from CPLEX, the average relative deviation is about $8.1 \%$. Regarding $\mathrm{LCH}$ performance on all instances, the average percentage relative difference is about $2.7 \%$.

\subsection{Results for the Port-au-Prince case study}

The Port-au-Prince graph after the 2010 earthquake has the following parameters: $|V|=16,657,|E|=19,558,|O|=4,|D|=62,|B|=536$, and $R=842$. Some simplifications on the graph could have been made, like the removal of nodes with degree one (that represents dead-end streets) which were not origins nor destination points. However, even with this pre-processing of the graph, it would still remain with a few thousand vertices and edges, and, therefore, unable to be handled by CPLEX. Hence, we decided to maintain the graph in its original form, keeping its coherence with the original data obtained by the satellite images.

The first accessibility estimation was performed applying Dijkstra's algorithm to the RNAP. Results indicated that only one destination was unreachable in the original graph, although SP to other destinations could still be improved by means of the proposed heuristics. $T$ was set to $\lceil R / Q\rceil=106$ time periods, ensuring that all edges could be repaired with $Q=8$ WT under the scenario assumed. A gradient map of the initial state of the road network is presented in Fig. 4. The larger the circles, the farther the node is from its nearest origin.

All the proposed heuristics were able to reduce the value of the weighted SP to all destinations $\left(\sum_{i \in D} p_{i} s_{i}^{t}\right)$ from 333 to 94,000 by the end of the first period (i.e., from $t=0$ to $t=1$ ). From the second period on, the changes in this value per time period are very small (less than $0.03 \%$. The heuristics $\mathrm{SH}, \mathrm{RH}$, and LCH consume, respectively, $43 \mathrm{~min}, 22 \mathrm{~s}$, and $25 \mathrm{~s}$, to allocate WT and evaluate accessibility for the first time period. To handle all 106 time periods, they require $13 \mathrm{~h}, 20 \mathrm{~min}$, and 4 min, respectively. Figure 5 presents the SP to destinations in the road network original state, where crossed roads are those to be repaired by WT according to the heuristics solution. 


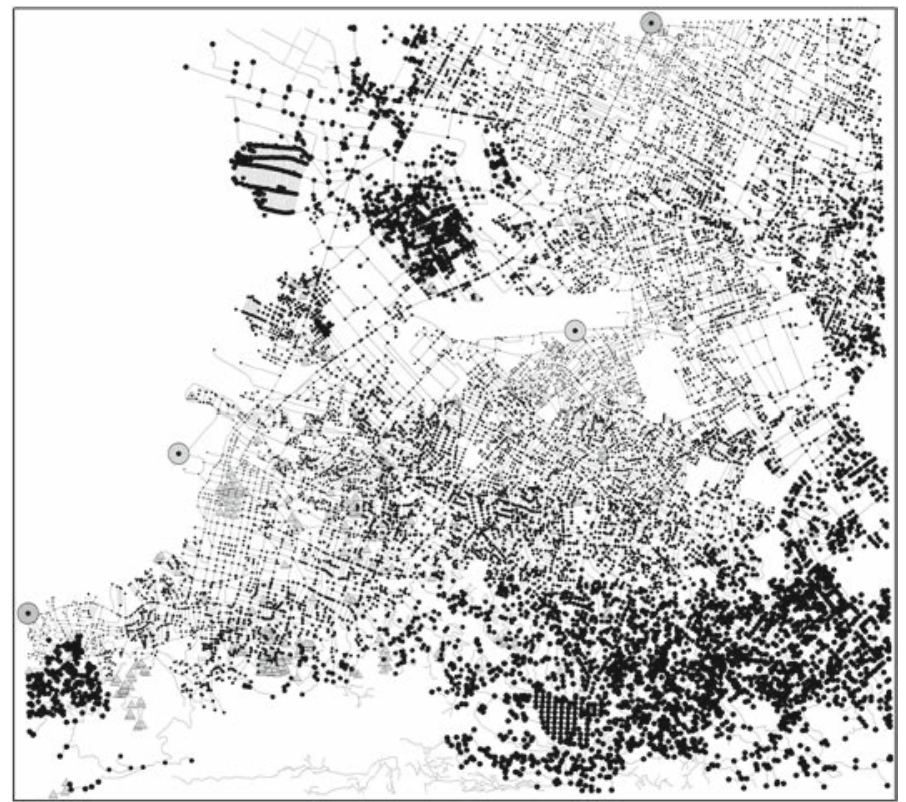

Fig. 4 Gradient map showing the initial accessibility of the road network

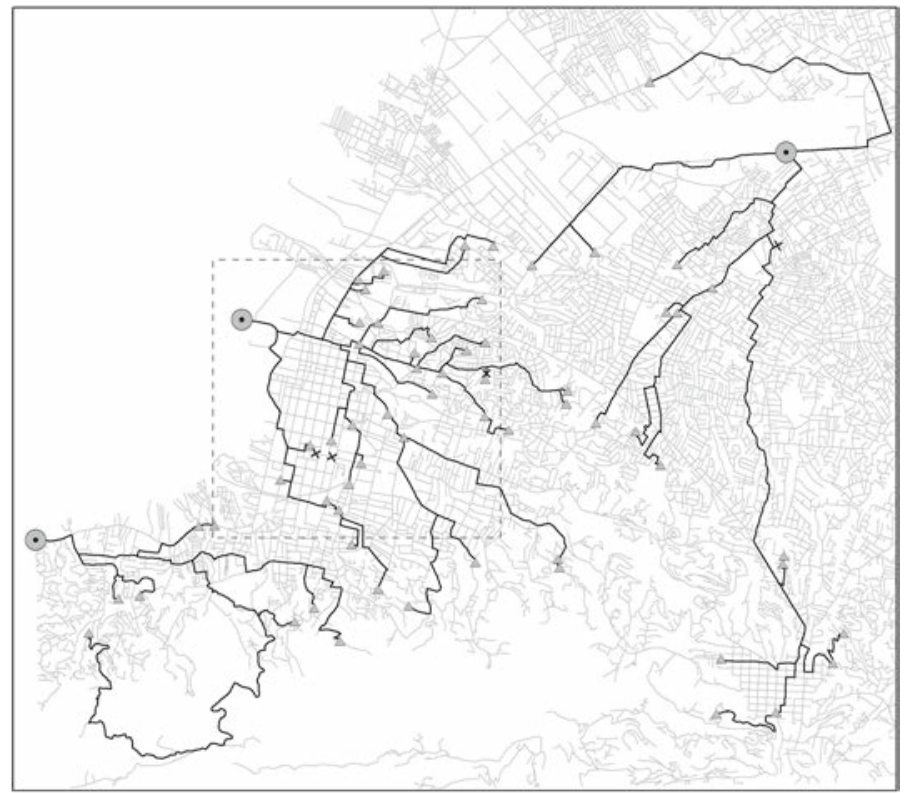

Fig. 5 SP from origins to destinations after the earthquake 


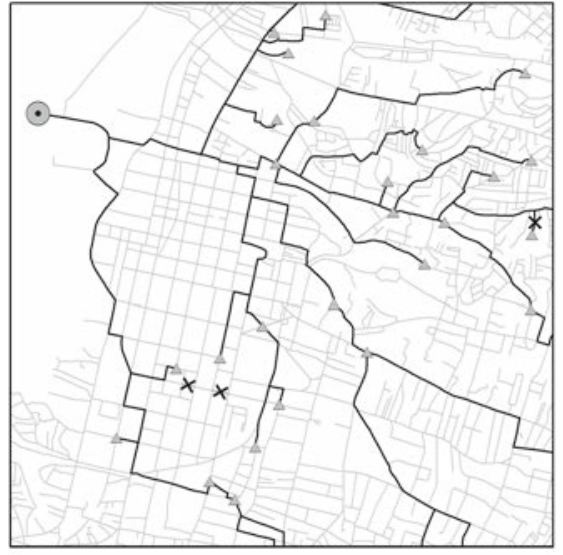

(a)

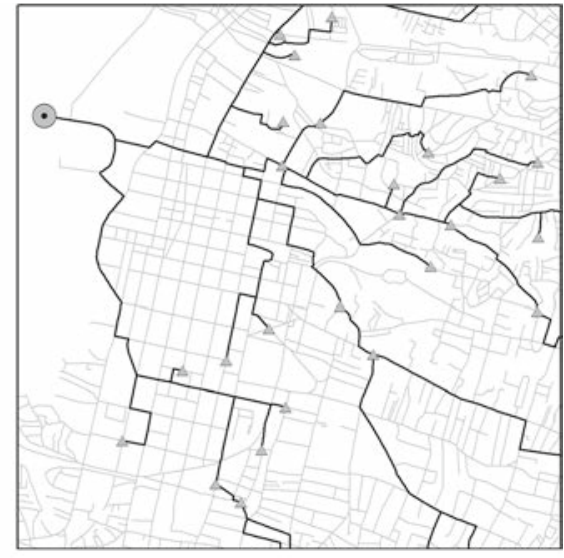

(b)

Fig. 6 SP in the area marked in Fig. 5 before (a) and after (b) first period repairs

It is important to mention that the time to allocate WT in the first time period plays a key role in real situations, since the algorithm has a whole time period to find the nextperiod allocation, once WT are sent. The processing times show that calculating the savings is a very time-consuming operation, making $\mathrm{SH}$ unfitted for large instances. $\mathrm{RH}$ and $\mathrm{LCH}$ used a similar amount of time to finish the first period allocation, but later on the smaller edge lists generated by $\mathrm{LCH}$ make the heuristic faster than $\mathrm{RH}$, whose edge ranking has a $O(|B|)$ size. These two heuristics present computational times acceptable for use in real operations.

Figure 6 exemplifies changes in the SP after the first period repairs in the area marked in Fig. 5. The three crossed roads in Fig. 6a are the ones repaired by WT during the first period. Figure 7 shows the accessibility improvement after WT first period repairs. The decrease in the SP to each node is proportional to the size of the dark circles, with the largest ones corresponding to a reduction of at least $200 \mathrm{~m}$. The dark square represents the originally unreachable destination that becomes accessible after edges selected by the heuristic are repaired in the first period.

From the results obtained, one can notice that even in cases when the most part of the population is already reachable after a major earthquake, the decision of where to send the WT first has an important role on the improvement of the accessibility to gathering camps. Another choice of roads to repair might have not granted access to one of the camps, and probably would not improve the accessibility to so many points as shown in Fig. 7. It is important to highlight that in such risky scenarios, the speed of relief teams is reduced. Thus, even small improvements in the traveling distances are significant.

\section{Concluding remarks}

In this work, we presented methods to deal with real instances of the Road Emergency Rehabilitation Problem after a major earthquake. Two mathematical models 


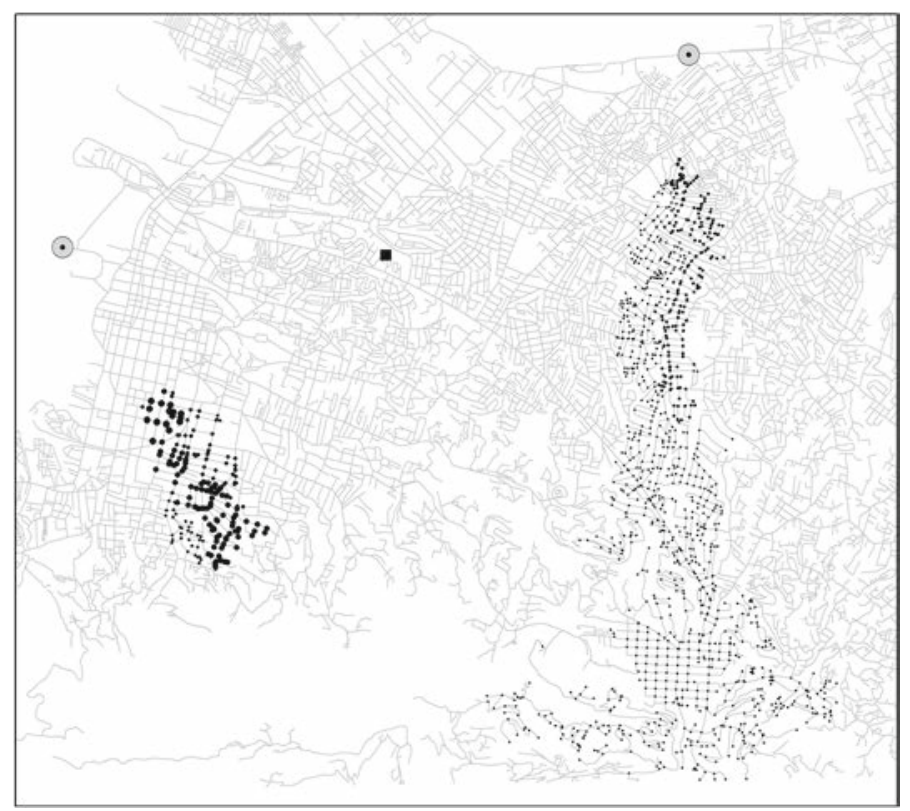

Fig. 7 Accessibility improvement after the first period repairs

for problems that compose the RERP are presented: the first one deals with the Road Network Accessibility Problem, allowing a decision maker to determine very quickly the accessibility of population gathering areas, by means of SP algorithms, such as Dijkstra. The second model concerns the Work-troops Scheduling Problem, generating WT allocations per time period to repair blocked edges of the damaged road network. Using both models, it is possible to know if the population is reachable after an earthquake and to improve their accessibility for relief teams from some origin points.

A critical point on applying optimization models for crisis management is data obtainment and treatment. A methodology for this purpose is described considering the initial data from satellite images and its conversion into graphs. The resulting graph of Port-au-Prince 2010 earthquake was used to test the performance of three heuristics for the WSP. The heuristics were initially tested on randomly generated instances with 10 vertices and 20 edges, for which CPLEX obtained optimal solutions for 72 out of 80 instances within $2 \mathrm{~h}$.

The experiments show that $\mathrm{LCH}$ is the best performance heuristic, with an overall average relative deviation of less than $3 \%$ from solutions obtained by CPLEX for simulated instances. It also performed well for the real instance, obtaining a first period WT allocation that reduces the sum of weighted SP to population gathering zones to less than one-third of its original value, in a few seconds. The other proposed heuristics are able to obtain similar good quality solutions for the WSP, improving population accessibility even for cases when all gathering areas are reachable from the beginning. 
Although LCH can quickly obtain good solutions for the WSP, the bottleneck of the decision making support tools lies today in the image obtaining and processing activities. Almost 2 days were past from the instant when the quake happened to the first road network damage evaluation. Automated faster methods of satellite image processing are necessary to make the developed tools effective after real disasters. However, since the problem can only be handled by any optimization method once all the image data are processed, $\mathrm{LCH}$ offers a reasonable time alternative to the use of optimization softwares for real scenarios, which would demand still hours to find a solution after the data are available.

The Port-au-Prince case study showed that the development of efficient tools guarantees accessibility improvements as fast as possible, which is crucial for relief operations. Even in cases when the population is reachable, the reduction of the traveled distances has a large impact on the efficiency of rescue teams.

The next steps of the OLIC project include the development of relief distributing algorithms associated with the road repairing schedule. In addition, long-term road network and city structures' rehabilitation are intended to be investigated as optimization problems applied to real situations, where a good planning may significantly reduce the time to reestablish a city struck by major disasters.

We hope that the introduction of a new model for the road rehabilitation problem after major disasters generates the interest of other researchers, with the development of different techniques for it as local searches, metaheuristics, and exact methods.

\section{References}

Aksu DT, Ozdamar L (2014) A mathematical model for post-disaster road restoration: enabling accessibility and evacuation. Transport Res Part E: Logist Transport Rev 61:56-67

Anaya-Arenas A, Renaud J, Ruiz A (2014) Relief distribution networks: a systematic review. Ann Oper Res 223(1):53-79

Bellman R (1958) On a routing problem. Q Appl Math 16:87-90

Caunhye AM, Nie X, Pokharel S (2012) Optimization models in emergency logistics: a literature review. Socio-Econ Plan Sci 46(1):4-13

Chen YW, Tzeng GH (1999) A fuzzy multi-objective model for reconstructing the post-quake road-network by genetic algorithm. Int J Fuzzy Syst 1(2):85-95

Copernicus Emergency Management Service (2016). http://emergency.copernicus.eu. Accessed 1 Jan 2015

Cormen TH, Stein C, Rivest RL, Leiserson CE (2009) Introduction to algorithms, 3rd edn. The MIT Press, Cambridge, USA

Diaz R, Behr J, Toba AL, Giles B, Ng M, Longo F, Nicoletti L (2013) Humanitarian/emergency logistics models: a state of the art overview. In: Proceedings of the 2013 summer computer simulation conference, Toronto, Canada, pp 1-24

Dijkstra EW (1959) A note on two problems in connexion with graphs. Numer Math 1(1):269-271

Duhamel C, Brasil D, Santos AC, Châtelet E, Birregah B (2014) Strategies to optimize the impact of supplies distribution in post-disaster operations. In: Proceedings of the 3rd international conference on operations research and enterprise systems, Angers, France, pp 342-349

Duque PM, Sorensen K (2011) A GRASP metaheuristic to improve accessibility after a disaster. OR Spectr 33(3):525-542

Feng CM, Wang TC (2003) Highway emergency rehabilitation scheduling in post-earthquake 72 hours. J East Asia Soc Transport Stud 5:3276-3285

Fiondella L (2013) An algorithm to prioritize road network restoration after a regional event. In: Proceedings of the IEEE international conference on technologies for homeland security, Waltham, USA, pp 19-25 
Giros A, Fontannaz D, Allenbach B, Treinsoutrot D, De Michele M (2012) Kal-haiti: a research database for risks management and sustainable reconstruction in haiti. In: ISPRS-international archives of the photogrammetry, remote sensing and spatial information sciences XXXIX-B8, pp 17-20

Google Earth (2016). https://earth.google.com. Accessed 1 Jan 2015

Hu Y, Liu X, Jiang Y (2012) Overviews of failure mode and reconstruction of road traffic facilities in wenchuan earthquak-stricken areas. Proc Environ Sci 12(A):615-627

International Charter "Space and Major Disasters" (2016). https://www.disasterscharter.org. Accessed 1 Feb 2014

Sakuraba CS, Santos AC, Prins C (2015) Modeling and solving the road network rehabilitation problem after a major earthquake. In: Proceedings of the 16th Congrès de la Société Française de Recherche Opérationnelle et Aide à la Décision. Marseille, France

Yan S, Lin CK, Chen SY (2012) Optimal scheduling of logistical support for an emergency roadway repair work schedule. Eng Optim 44(9):1035-1055

Yan S, Chu JC, Shih YL (2014) Optimal scheduling for highway emergency repairs under large-scale supply. IEEE Trans Intell Transport Syst 16(6):2378-2393

Yan S, Shih YL (2007) A time-space network model for work team scheduling after a major disaster. J Chin Inst Eng 30(1):63-75

Yan S, Shih YL (2009) Optimal scheduling of emergency roadway repair and subsequent relief distribution. Comput Oper Res 36(6):2049-2065 